\title{
Fase crônica da COVID-19: desafios do fisioterapeuta diante das disfunções musculoesqueléticas
}

\author{
Chronic phase of COVID-19: challenges for physical therapists \\ in the face of musculoskeletal disorders
}

O novo coronavírus (SARS-CoV-2) faz parte de um grupo de vírus responsáveis por causar síndromes respiratórias agudas que podem variar de sintomas leves a condições graves, com internação hospitalar, necessidade de ventilação mecânica e significativa taxa de mortalidade [1]. Apesar do comprometimento respiratório, outros sintomas sistêmicos podem ser manifestos, tais como distúrbios neurológicos, gastrointestinais e musculoesqueléticos [2].

Embora pouco seja conhecido sobre as consequências físicas da COVID-19 a longo prazo, os pacientes que necessitam de ventilação mecânica na fase mais aguda da doença podem vivenciar sérios efeitos colaterais, desenvolvendo a chamada síndrome pós-cuidados intensivos, que acomete sobreviventes de todas as idades. Essa síndrome é caracterizada primariamente por uma incapacidade prolongada e tem como efeitos secundários disfunção muscular, fadiga, dor e dispneia [3]. Uma segunda consequência muito comum nos pacientes graves consiste em fraqueza adquirida na UTI, relacionada à imobilidade, controle glicêmico abaixo do ideal e iatrogenia pelo uso de esteroides e bloqueadores neuromusculares. Outras possíveis alterações subsequentes são a polineuropatia e a miopatia do paciente crítico. Podem ocorrer ainda sequelas físicas menos comuns, decorrentes da imobilidade prolongada, incluindo descondicionamento cardiorrespiratório, instabilidade postural, tromboembolismo venoso, encurtamento muscular, contraturas (miogênicas, neurogênicas, artrogênicas) e úlceras por pressão [4].

A fim de limitar a gravidade de todas as sequelas decorrentes do processo de internação, é essencial a atuação do fisioterapeuta ainda no ambiente hospitalar, na fase mais precoce da doença, o que vai promover uma recuperação funcional mais rápida e acelerar o processo de alta. Em alguns casos, nos quais a infecção gera tosse produtiva, o fisioterapeuta conduzirá técnicas de higiene brônquica que permitirão a eliminação das secreções e ajudarão a diminuir o desconforto respiratório. Em outras situações nas quais haja uma tosse seca e não produtiva, o que é bem mais frequente mediante a COVID-19, a fisioterapia respiratória pode não ser necessária [5]. No entanto, considerando que o papel do fisioterapeuta na fase aguda da doença não se restringe ao sistema respiratório, esse profissional permanece indispensável nessa fase, conduzindo exercícios e mobilizações que minimizarão significativamente os déficits musculoesqueléticos decorrentes do imobilismo prolongado.

Nos pacientes não graves, a doença também pode gerar consequências duradouras. Como ainda não foi descoberto um tratamento eficaz no combate direto ao vírus, além dos cuidados de higiene, o isolamento 
social ainda é a opção mais recomendada pela Organização Mundial de Saúde a fim de conter a alta velocidade de contaminação e minimizar a sobrecarga nos sistemas de saúde. Em contrapartida, essa medida pode acentuar ainda mais uma outra pandemia com a qual o mundo já convive há muitos anos: a inatividade física e o sedentarismo [6].

Mesmo sem uma relação com a COVID-19, o isolamento social é um fator contribuinte para o aparecimento de sintomas musculoesqueléticos, tais como dor miofascial e artralgias, principalmente aquelas ligadas às doenças autoimunes como artrite reumatoide, espondilites e lúpus eritematoso sistêmico. Por intensificar a ansiedade e o estresse, a restrição também pode aumentar a sintomatologia de pacientes com fibromialgia [7 - 9]. Nesse contexto, o fisioterapeuta que atua nas disfunções musculoesqueléticas se depara com duas complicações: pacientes que já apresentavam limitações físicas tendo que lidar com a redução da quantidade de exercícios e a minimização dos recursos fisioterapêuticos [10], e a imprevisível resposta de pacientes recuperados de COVID-19 a médio e longo prazo.

A fim de minimizar tais impactos, os conselhos profissionais da fisioterapia no Brasil liberaram os serviços de teleconsultas, teleconsultoria e telemonitoramento como ferramentas aplicáveis e reprodutíveis para permitir a supervisão e atenção aos pacientes que necessitam de intervenção clínica [11]. A atividade física diminui o estresse, melhora a autoestima, a capacidade cardiorrespiratória, a força muscular e a coordenação, previne a fragilidade, a sarcopenia e a dinapenia, além de minimizar o risco de quedas e o declínio cognitivo em idosos [12]. O monitoramento de exercícios, as orientações posturais e funcionais, bem como o alerta aos pacientes com doenças crônicas sobre o respeito aos princípios de conservação de energia, podem ser recursos fundamentais para evitar a eclosão de um estado de crise desses pacientes, bem como o surgimento de sintomas antes não apresentados [13]. Nesse sentido, os profissionais de reabilitação têm um papel fundamental no período de isolamento, ajudando a otimizar a independência funcional e melhorar a qualidade de vida, além de facilitarem uma posterior reintegração comunitária [4].

Estudos realizados com pacientes que apresentaram a SARS (severe acute respiratory syndrome) causada pela forma mais antiga de coronavírus (SARS-CoV) mostraram redução da capacidade cardiorrespiratória, limitação musculoesquelética e redução da qualidade de vida mesmo após o término da doença. Isso mostra a necessidade de recuperação desses pacientes quanto à sua capacidade funcional [14]. Os indivíduos apresentavam elevados níveis de estresse, depressão e ansiedade mesmo um ano após a sobrevivência. Outras doenças virais que ocasionam SARS são descritas na literatura como geradoras de incapacidade pulmonar a longo prazo e comprometimento psicológico mesmo dois anos após a alta. Os estudiosos alertam que "os efeitos a longo prazo da doença infecciosa não devem ser ignorados" [15].

Para a melhoria desses sintomas, é importante o monitoramento realizado pelo fisioterapeuta, bem como um programa intensivo de reabilitação física proposto para esses pacientes, com períodos variáveis de 6 meses a 2 anos. Analisando a realidade manifestada em pacientes com COVID-19, percebe-se que os mesmos, ao cursarem com SARS, podem apresentar uma necessidade de suporte terapêutico também em fases crônicas ou após a cura da doença [12].

Assim, conclui-se que uma nova esfera de acompanhamento clínico desses pacientes é despertada para os profissionais de fisioterapia. A necessidade de promover o retorno à plena funcionalidade de pacientes curados da COVID-19, bem como a recuperação física da população em isolamento, prometem ser demandas crescentes, que irão requerer dos fisioterapeutas que atuam fora do ambiente hospitalar uma busca pelo aprimoramento e recuperação dos pacientes que surgirão nos serviços de saúde após os primeiros ciclos da pandemia ocasionada pelo atual coronavírus.

\section{Rodrigo Marcel Valentim da Silva (10 ${ }^{[a]}$, Angelica Vieira Cavalcanti de Sousa $\mathbb{( i b}^{[b]}$}

\footnotetext{
[a] Doutor em Fisioterapia, Universidade Federal do Rio Grande do Norte (UFRN), Natal, RN, Brasil

${ }^{[\mathrm{b}]}$ Doutoranda em Ciências da Saúde, Charité Universitätsmedizin, Berlim, Alemanha
} 


\section{Referências}

1. Rothan HA, Byrareddy SN. The epidemiology and pathogenesis of coronavirus disease (COVID-19) outbreak. J Autoimmun. 2020;109:102433.

2. Nicola M, Alsafi Z, Sohrabi C, Kerwan A, Al-Jabir A, Iosifidis $\mathrm{C}$, et al. The socio-economic implications of the coronavirus and COVID-19 pandemic: a review. Int J Surg. 2020. doi: 10.1016/j.ijsu.2020.04.018 [Epub ahead of print]

3. Falvey JR, Krafft C, Kornetti D. The essential role of home- and community-based physical therapists during the COVID-19 pandemic. Phys Ther. 2020. pii:pzaa069. doi: 10.1093/ptj/pzaa069 [Epub ahead of print]

4. Simpson R, Robinson L. Rehabilitation following critical illness in people with COVID-19 infection. Am J Phys Med Rehabil. 2020. doi: 10.1093/ptj/pzaa069 [Epub ahead of print]

5. Thomas P, Baldwin C, Bissett B, Boden I, Gosselink $\mathrm{R}$, Granger $\mathrm{CL}$, et al. Physiotherapy management for COVID-19 in the acute hospital setting: clinical practice recommendations. J Physiother. 2020;66(2):73-82.

6. Hall G, Laddu DR, Phillips SA, Lavie CJ, Arena R. A tale of two pandemics: how will COVID-19 and global trends in physical inactivity and sedentary behavior affect one another? Prog Cardiovasc Dis. 2020. doi: 10.1016/j. pcad.2020.04.005 [Epub ahead of print]

7. Misra DP, Agarwal V, Gasparyan AY, Zimba 0 . Rheumatologists' perspective on coronavirus disease 19 (COVID-19) and potential therapeutic targets. Clin Rheumatol. 2020. doi: 10.1007/s10067-020-05073-9 [Epub ahead of print]

8. Li W, Yang Y, Liu ZH, Zhao YJ, Zhang Q Zhang L, et al. Progression of Mental Health Services during the COVID-19 Outbreak in China. Int J Biol Sci. 2020;16(10):1732-8.
9. Haines KJ, Berney S. Physiotherapists during COVID-19: usual business, in unusual times. J Physiother. 2020;66(2):67-9.

10. Hamid S, Mir MY, Rohela GK. Novel coronavirus disease (COVID-19): a pandemic (epidemiology, pathogenesis and potential therapeutics). New Microbes New Infect. 2020;35:100679.

11. Conselho Federal de Fisioterapia e Terapia Ocupacional (COFFITO). Resolução n ${ }^{\circ}$ 516, de 20 de março de 2020 Teleconsulta, Telemonitoramento e Teleconsultoria [acesso 30 abr 2020]. Disponível em: https://www.coffito.gov.br / nsite/?p=15825

12. Jiméne-Pavón D, Carbonell-Baeza A, Lavie CJ. Physical exercise as therapy to fight against the mental and physical consequences of COVID-19 quarantine: special focus in older people. Prog Cardiovasc Dis. 2020. doi: 10.1016/j. pcad.2020.03.009 [Epub ahead of print]

13. Fallon K. Exercise in the time of COVID-19. Aust J Gen Pract. 2020;49. doi: 10.31128/AJGP-COVID-13 [Epub ahead of print]

14. Hui DS, Wong KT, Antonio GE, Tong M, Chan DP, Sung JJ. Long-term sequelae of SARS: physical, neuropsychiatric, and quality-of-life assessment. Hong Kong Med J. 2009;15 (Suppl 8):S21-3.

15. Lau HM, Lee EW, Wong CN, Ng GY, Jones AY, Hui DS. The impact of severe acute respiratory syndrome on the physical profile and quality of life. Arch Phys Med Rehabil. 2005;86(6):1134-40. 